\title{
A STUDY OF THE DIVERSE RACES OF ENT- AMOEBA HISTOLYTICA DISTINGUISHABLE FROM ONE ANOTHER BY THE DIMENSIONS OF THEIR CYSTS.
}

\author{
BY CLIFFORD DOBELL \\ (Imperial College of Science)
}

AND MARGARET W. JEPPS

(Bathurst Student, Newnham College, Cambridge).

(With 7 Text-figures and Plate VI.)

(A REPORT TO THE MEDICAL RESEARCH COMMITTEE ${ }^{1}$ )

CONTENTS.

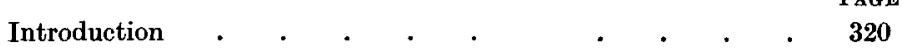

Part I.

A. Methods used to determine the dimensions of the cysts

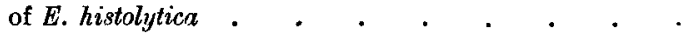

B. Determination of the differences in the dimensions of cysts when examined alive and after fixing, staining, and mounting: with an inquiry into the factors which determine these differences . . . . . .

Part II.

Demonstration of the existence of races of $E$. histolytica which differ from one another in the size of the cysts which they produce. . . . . . . . 334

Comparison of our conclusions with those of some other workers 344

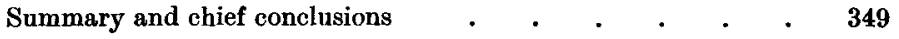

References . . . . . . . . . . . 350

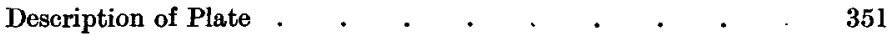

\section{INTRODUCTION.}

IN a recent paper (1917) we have drawn attention to the fact that there is great variation in the size of the cysts formed by the dysentery amoeba,

1 The work here described was chiefly carried out, with the aid of grants from the Medical Research Committee, at the Wellcome Bureau of Scientific Research. We wish to record our indebtedness once more to the Bureau, for its unfailing help, and for its hospitality whilst the work was in progress. 
Entamoeba histolytica ${ }^{1}$. We stated that there are different races or strains of this species distinguishable from one another by the dimensions of their cysts, our conclusions in this respect agreeing closely with those of Wenyon and O'Connor (1917). In the following pages we propose to present in greater detail the evidence for our conclusions. Before doing so, however, it is necessary to enter into some detail concerning certain preliminary investigations which were indispensable for a proper demonstration of this fact. Therefore, we shall first consider these necessary preliminaries; then present our evidence and the conclusions which we have drawn from it; and finally discuss the results of some other observers in the light of our own findings.

\section{Methods AND Results.}

Part I.

\section{A. Methods used to determine the dimensions of the cysts of} E. histolytica.

An exact determination of the dimensions of the cysts of $E$. histolytica is by no means as easy as it might at first sight appear. The cysts are of small size, seldom perfectly symmetrical, and subject to considerable modification in various respects by the medium in which they are measured. It is moreover necessary to make a large number of measurements before any definite conclusion can be drawn from them. We therefore think it important to give in detail the methods we have adopted for this investigation.

When the cysts are carefully examined under the microscope it is found that very few indeed are perfectly spherical ${ }^{2}$. Even these which at first sight appear to be so, will usually be found not truly spherical if moved and viewed from other aspects. If we were to confine our attention

1 We continue to use this name, rather than $E$. dysenteriae, in referring to this organism, for reasons given elsewhere by one of us (Dobell, 1918).

2 Cf. the outlines of cysts shown in the figures on Plate VI. We have seen it stated several times that the living cysts of $E$. histolytica are in reality perfectly spherical, and that fixed and stained cysts of different shape have been produced artificially in making the preparations. This may be the rule when the technique employed has been bad; but we find it hard to believe that anybody who has carefully studied a large number of cysts, both living and stained, and in many different infections, can uphold such an opinion. Most living eysts are not perfectly symmetrical; and obviously asymmetrical living eysts are found commonly in most infections and in some almost exclusively. Although, as will be shown in experiments recorded below, fixation-even with a good fixative - may alter the shape of a cyst, it does not appreciably modify the majority: and we shall show that, when fixation does change the shape of a cyst, this change may actually be from an oval to a spherical shape. 
therefore to those cysts which are perfectly spherical, and thus capable of exact and rapid measurement, we should ignore the vast majority of cysts in any infection; a procedure which might lead to a totally false estimate of the dimensions of the cyst population as a whole. Accordingly we have ignored only those cysts which were much misshapen and which formed only a very small proportion of the total number in the infections here described; and we have measured all those which were approximately elliptical or circular in outline. The size of the latter was recorded simply as the diameter, and of the former as the mean of the longer and shorter axes.

All the measurements were made with a finely-ruled ocular micrometer, in combination with a $1 / 12^{\prime \prime}$ oil immersion objective. The microscope was adjusted in all cases so that one division of the micrometer scale represented $1.5 \mu$. We always estimated the diameter of a cyst to the nearest half of a division, so that the unit employed was in reality

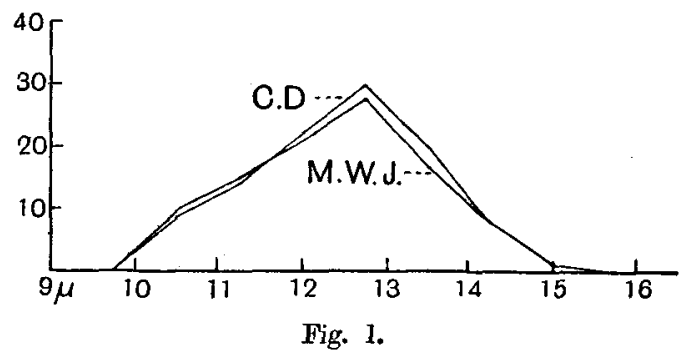

$0.75 \mu$. All the measurements were recorded directly in units of the micrometer scale, and not converted into microns until afterwards. Perfectly accurate measurements cannot of course be obtained by this method, but we know of none better for obtaining a large series of fairly trustworthy measurements within a reasonable time.

Since every measurement made in this manner involves a certain amount of estimation on the part of the measurer, it is clear that the results recorded by different individuals are likely to differ slightly from one another. We endeavoured to determine our own individual differences in this respect in the following way. One hundred cysts in a fixed and stained preparation were measured as carefully as possible, each cyst being measured by each of us independently, and the measurements independently recorded. On comparing the results we found that the dimensions registered were identical for 71 of the cysts. For each of the remaining 29 cysts there was a difference of half a micrometer division, 
or $0.75 \mu$, between the two measurements. One of us (M. W. J.) recorded the diameters of 18 of these 29 cysts as half a division smaller, and 11 as half a division larger, than the other (C. D.). The two series of measurements when recorded graphically gave curves of closely similar form (Fig. 1). We concluded therefore that for the majority of cysts measured our findings would be identical, whilst in the case of the remainder the individual error would not be greater than one unit of the scale employed: and furthermore that this error would probably not influence the character of our curves. We decided to measure a large number of cysts (500) in every infection studied, in order to obtain an approximately true average value for the sizes of the cysts; and to measure half (250) of this number each in every 500 , so as to eliminate as far as possible the personal error. Each series of measurements here recorded was obtained in this way.

As we recorded all our measurements in units of the micrometer scale we found it most convenient to plot our curves in these terms. The conversion of the units into microns, as shown in the charts, was made directly on the charts themselves. For this reason the points on the curves do not always coincide with the integral values in microns on the abscissae.

B. Determination of the differences in the dimensions of cysts when examined alive and after fixing, staining, and mounting: with an inquiry into the factors which determine these differences.

It is well known that the dimensions of cysts after fixation, staining, and mounting are not the same as those of the living cysts. As, however, it was not always possible to measure large numbers of living cysts at the time when they were obtained, it became necessary to rely upon fixed and stained material; and it therefore became necessary also to ascertain what effect this would have on our measurements. To determine this point we adopted two different methods: first, the measurement of a large number of cysts (from the same infection) both living and when fixed, stained, and mounted-the mean dimensions so obtained being then compared; and secondly, we devised a method for observing and measuring an individual cyst during the various processes of fixation, staining, and mounting in balsam. Throughout this paper the expression "fixed, stained and mounted in balsam" signifies-unless the contrary is stated-that fixation was effected with a modified form of Schaudinn's fluid (saturated corrosive sublimate in water, two parts; absolute 
alcohol, one part; glacial acetic acid, 4-5\%), and staining with Mayer's haemalum; and that the mounting medium was Canada balsam dissolved in xylol. This procedure was adopted because it was that which, after a long series of trials of various methods ${ }^{1}$ made by one of us (C. D.), was found to give the most reliable, uniform, and rapid results in practice. We have long used this, therefore, as a routine method. Cysts fixed in this manner are not as a rule appreciably shrunken or distorted; and although the stain is inferior in some respects to iron-haematoxylin and some other methods, it permits of the study of considerable cytological detail, whilst demanding but a small fraction of the time, labour, and attention requisite for these.

The cysts selected for the first method of investigation were obtained from a convalescent dysentery patient (E. 42), who had long been under observation, and whose cysts were of a conveniently large size. From a stool passed by this patient we measured

(1) 500 living cysts in $0.75 \%$ saline solution;

(2) 500 cysts in Weigert's iodine solution;

(3) 500 cysts fixed, stained, and mounted in Canada balsam;

(4) 500 cysts similarly fixed and stained, but mounted in Gilson's euparal.

Our results are given in the following Table (Table I) and recorded graphically in Fig. 2.

\section{TABle $\mathrm{I}$.}

Dimensions of cysts in units of ocular micrometer*.

\begin{tabular}{|c|c|c|c|c|c|c|c|c|c|c|c|c|c|c|}
\hline & & & $5 \cdot 5$ & $6 \cdot 0$ & $6 \cdot 5$ & $7 \cdot 0$ & $7 \cdot 5$ & $8 \cdot 0$ & 8.5 & $9 \cdot 0$ & $9 \cdot 5$ & $10 \cdot 0$ & $10 \cdot 5$ & $11 \cdot 0$ \\
\hline \multicolumn{3}{|c|}{ No. of cysts in saline } & - & - & - & 7 & 28 & 130 & 108 & 136 & 52 & 32 & 3 & 4 \\
\hline " & $"$ & iodine & - & - & - & 16 & 29 & 117 & 126 & 134 & 45 & 26 & 7 & - \\
\hline צ' & " & balsam & 1 & 0 & 9 & 60 & 90 & 165 & 101 & 54 & 12 & 8 & - & - \\
\hline & ," & euparal & - & 1 & 3 & 49 & 86 & 166 & 101 & 67 & 21 & 5 & 1 & 一 \\
\hline
\end{tabular}

* As explained above, 1 micrometer division $=1.5 \mu$.

1 In the course of this work it was found that the majority of the ordinary cytological fixatives give inconstant results with $E$. histolytica cysts, though many are at times excellent. Schaudinn's fluid, for example (without acetic acid), frequently gives results quite as good as those obtained with the liquid mentioned above. Its action, however, is less uniform. It appears immaterial whether $4 \%$ or $5 \%$ of glacial acetic be added, but the addition of either more or less gave less satisfactory results. The only other fixative which gave equally uniform and excellent fixation was a mixture consisting of four parts of saturated picric acid in $90 \%$ alcohol and one part of glacial acetic acid. The only objection to the use of this fluid for routine work is the length of time necessary to wash out the picric acid before staining. 
The mean sizes of the cysts calculated from these figures and expressed in microns are as follows:

Mean size of cysts in saline $\quad=12.99 \mu$ or approximately $13 \mu$

$\begin{array}{lllll}" & \quad \text { iodine }=12 \cdot 86 \mu, & & 12 \cdot 9 \mu \\ " & \quad & \text { balsam }=12 \cdot 04 \mu " & & 12 \mu \\ " & \quad & \text { euparal }=12 \cdot 19 \mu, & & 12 \cdot 2 \mu \text {. }\end{array}$

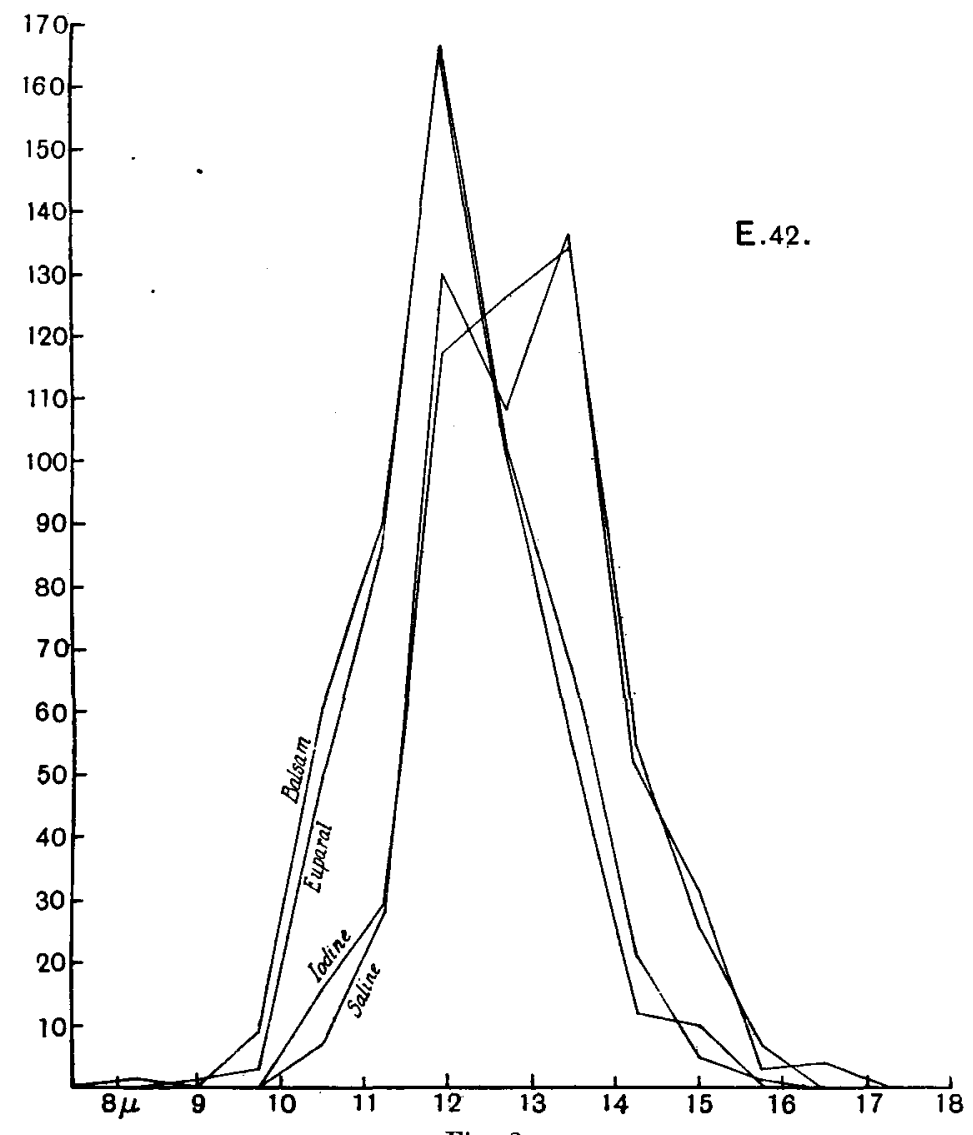

Fig. 2.

The differences in the dimensions of the cysts measured in different media are very clearly shown by the curves ${ }^{1}$ (Fig. 2). It will be noted that the curves representing the measurements of the cysts in euparal and balsam coincide very nearly, each having a single apex at $12 \mu$. The

1 In these curves, and in all the others in this paper, the numbers of cysts measured are shown on the ordinates, and the dimensions of the cysts in microns along the abscissae. 
curves for the cysts in saline and iodine are also to a great extent superimposed; but whilst the latter possesses a single summit at $13.5 \mu$, the former displays two apices situated at $12 \mu$ and $13 \cdot 5 \mu$, although numerous cysts of intermediate size are also recorded.

The curve obtained from the measurements in saline was so curiously different in character-having two apices instead of one-that we were at a loss to account for it. It occurred to us that a possible explanation might be that, in measuring the cysts, we had unconsciously tended to estimate the dimensions to the nearest line on the micrometer scale $(12 \mu$ and $13.5 \mu$ represent respectively eight and nine divisions of the scale): and that we might thus have distributed numerous cysts of intermediate size to those categories immediately above and below them. We decided therefore to make another series of measurements from the same case. Accordingly, a month later, we obtained another sample of faeces from this patient, and measured another $500 \mathrm{cysts}$ in saline. The results were as follows:

\section{TABLE II.}

\begin{tabular}{ccccccccccc} 
& \multicolumn{8}{c}{ Dimensions of cysts in units of ocular micrometer. } \\
\cline { 2 - 9 } & $7 \cdot 0$ & $7 \cdot 5$ & $8 \cdot 0$ & $8 \cdot 5$ & $9 \cdot 0$ & $9 \cdot 5$ & $10 \cdot 0$ & $10 \cdot 5$ & $11 \cdot 0$ \\
No. of cysts & 7 & 15 & 90 & 133 & 121 & 74 & 45 & 10 & 5
\end{tabular}

These figures, when presented graphically (Fig. 3), give a curve with a single summit like the others-the summit in this case being at $12.75 \mu$ or midway between the two summits of the first saline curve (Fig. 2). As we have never at any other time obtained a curve of the latter type from a series of measurements of $E$. histolytica cysts, we believe that in this case it was an accidental result of factors which we cannot at present specify with precision, though we believe the explanation suggested above is probably correct.

On comparing the average dimensions of the cysts measured in different media, it will be seen that the size of those measured in saline and iodine is approximately the same $(12.99 \mu$ and $12.86 \mu)$, whilst that of those measured in balsam is less $(12 \cdot 04 \mu)$. We concluded therefore that the action of iodine has no effect upon the size of the cysts-neither shrinking them nor causing them to swell-when used as a fixative: but that the apparent size after fixing, staining, and mounting in balsam is appreciably diminished. This diminution in average size amounted in the present case to approximately $1 \mu$.

To what is this apparent shrinkage due? A careful study of the cysts in saline and balsam seemed to us to indicate quite clearly that it is due 
merely to the circumstance that the cyst wall is clearly visible in the former medium, but almost always invisible in the latter. The diameter of a cyst measured in saline therefore includes twice the thickness of the cyst wall, whereas that of a cyst mounted in balsam is really the diameter of the stained protoplasmic contents only. This supposition is supported by the measurements made of cysts in euparal. As is well known, the refractive index of euparal is lower than that of Canada balsam dissolved in xylol; and accordingly the walls of cysts mounted in the former medium are frequently visible, and would therefore be often included in measurements of their diameters. We should therefore expect that the mean size of cysts mounted in euparal would be intermediate between

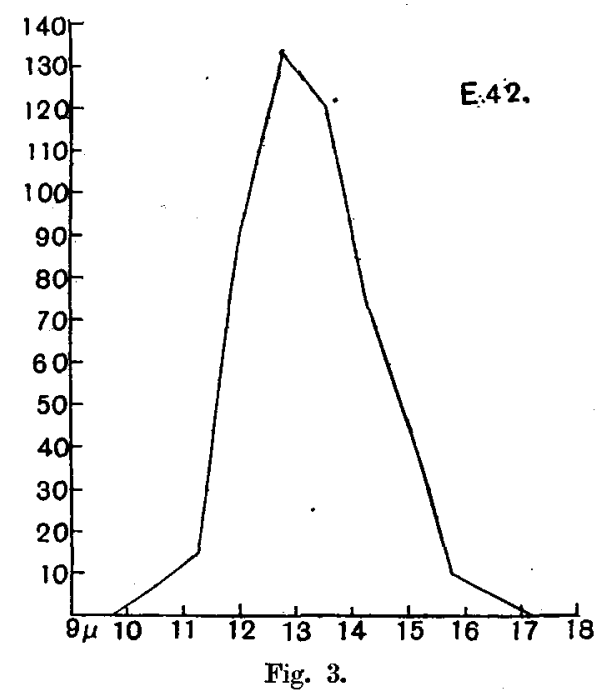

the sizes of those in saline and those in balsam. This we found to be the case, the mean size of cysts in euparal being $12 \cdot 2 \mu$. If this explanation is correct we should expect further that the cysts actually measured in balsam would, if remounted in a medium of lower refractive index, be found to have a greater mean size. To test this we remounted in water the coverglasses which had been originally mounted in balsam, and from which the above measurements had been made. Five hundred cysts were again measured in them, and the mean size was found to be $12 \cdot 3 \mu$ as against $12.04 \mu$ for the same cysts in balsam. This increase in size, though appreciable, was not so great as we had expected: but we considered that the measurements of the cysts in water were not altogether trustworthy. It was often extremely difficult, if not impossible, to distinguish 
the outline of the cyst wall sharply, on account of the opacity of the stained cysts and the surrounding bodies in the preparation.

More direct evidence was obtained by measurements of the thickness of the wall of individual cysts. The cyst wall of $E$. histolytica is extremely thin, and consequently difficult to measure. We were able, however, to obtain some satisfactory measurements by means of the camera lucida. The cysts were studied under a $2 \mathrm{~mm}$, apochromat (N.A. $=1 \cdot 40$ ), with compensating ocular 18, and with critical illumination from an achromatic aplanatic condenser $(N . A .=1 \cdot 40)$, the measurements being read off directly from the projected image at a magnification of 2500 diameters. The thickness of the cyst wall was measured in living cysts, in cysts plasmolysed in $10 \%$ sodium chloride solution, and in fixed, unstained cysts suspended in $70 \%$ alcohol. The thickness of the cyst wall was found to be remarkably uniform. In no case, after making between 30 and 40 measurements, was it found to be less than $0.5 \mu$ or greater than $0 \cdot 6 \mu$. The mean value for 20 such measurements was $0.506 \mu$. It should be added that the cysts used for these measurements were of approximately the same size as those from case E. 42 , but were obtained from two different patients.

These figures are in complete agreement therefore with the supposition made above, namely, that the decrease in size observable in cysts mounted in balsam is due chiefly to the invisibility of the cyst walls in this medium. If the thickness of the cyst wall is $0.5-0.6 \mu$, we should expect the diameters of cysts in saline or iodine-in which the cyst walls are visible - to be on an average greater by twice this amount $(1 \mu-1 \cdot 2 \mu)$ than the diameter of those in balsam-in which the cyst walls are not visible. And, as we have already stated, the mean diameter of cysts in balsam was actually found to be approximately $1 \mu$ less than that of cysts measured in saline or iodine solution.

It is clear that a direct proof of the correctness of this interpretation could be obtained if individual cysts could be studied microscopically both when alive, and during all the processes of fixation, staining, and mounting. As noted above, we succeeded, after some failures, in devising a simple method of doing so. Our procedure is as follows. A small sample of faeces containing cysts of $E$. histolytica is mixed on a slide with a suitable quantity of gelatine jelly previously liquefied by warming. Before the gelatine has time to set, a small drop of this emulsion is spread in the middle of a coverglass $\left(7 / 8^{\prime \prime} \times 7 / 8^{\prime \prime}\right)$, and the latter placed inverted in the concavity of a hollowground glass block $(6 \mathrm{~cm} . \times 6 \mathrm{~cm} ., 1 \cdot 25 \mathrm{~cm}$. thick, with a deep concavity $5 \mathrm{~cm}$. in diameter). The coverglass thus 
stands on its four corners in the middle of the concavity, with its film downwards. The corners are then firmly cemented to the block with drops of paraffin wax. The preparation is then placed on the stage of the microscope and the film examined under a low power. When a cyst of $E$. histolytica has been found, it is carefully placed under the oil immersion in the centre of the field, and measured with the ocular micrometer. When the gelatine has set, the fixative is introduced with a fine pipette under the coverglass, and allowed to fill completely the space between it and the block. When this operation is properly performed, the cyst, which is observed continuously during the process, will be found to remain in position and not to be dislodged by the entry of the fixative. Its measurements after fixation may then be again recorded.

In carrying out the above process, the following points should be kept in mind, since the success of the experiment depends upon them. The gelatine solution used must be of such concentration that it will liquefy at a very low temperature (so as not to injure the cysts) but set at the ordinary temperature of the laboratory. The concentration of salts in it must also be such that living cysts are unaffected by it. We found a $5 \%$ solution of gelatine in physiological saline solution $(0.75 \%$ sodium chloride in distilled water) to fulfil these requirements perfectly. Cysts in this medium appear perfectly normal under the microscope, and remain, in both the sol and the gel, unstainable with eosin (Kuenen and Swellengrebel's test of the vitality of cysts). We tried a jelly deeply coloured with eosin in the expectation that the cysts would be more sharply defined, and therefore easier to measure, in this medium. We found, however, that the colourless jelly is preferable. The film must be made of a suitable thickness, which can only be determined by a few trials. It must not be so thick as to prevent exact measurement of the cyst after fixation of the jelly, nor so thin that the gelatine rapidly undergoes desiccation after it is set. The fixative must be introduced when the gelatine has completely set, but before it has had time to dry. The setting of the gelatine can easily be determined under the microscope by observing the complete cessation of Brownian movement in the smallest particles in the preparation.

We made a number of observations by this method, and give below the results in tabular form (see Table III). The fixative used in all these experiments was that described above (sublimate-alcohol-acetic-acid). In the table the first measurement given for each cyst is the vertical diameter, the second the horizontal. Fixation of the cysts as seen under the microscope was almost instantaneous. As a rule the measurements 
after fixation were made within five minutes of the application of the fixative, but in some cases they were repeated at intervals up to one and a half hours later. When this was done we found the prolonged action of the fixative produced no subsequent change in size. The measurements in every case were made by one of us and checked by the other before being recorded. The cysts were from an infection in which they were of large size, and thus convenient for measuring.

Table III.

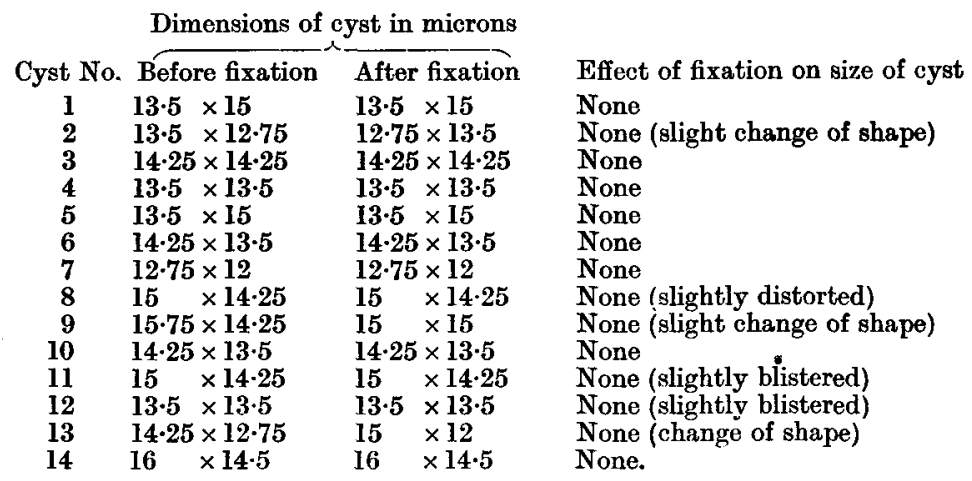

From these experiments we conclude that living cysts, when fixed with the solution which we have used, undergo no change in size whatever as the result of fixation.

The effects of staining, dehydration, clearing, and mounting in balsam were studied under the microscope in the same way as the effects of fixation: that is to say, the fixative was removed with the pipette, and other reagents substituted successively as required. We give below the results of three such experiments in detail.

Expt. 1. (Cyst No. 4 of Table III).

\begin{tabular}{|c|c|c|c|}
\hline \multicolumn{3}{|l|}{ Medium in which cyst was examined } & Dimensions \\
\hline Gelatine jelly (living cyst) & $\ldots$ & ... & $13.5 \mu \times 13.5 \mu$ \\
\hline Fixative (after 5 mins.) & $\ldots$ & $\ldots$ & $13.5 \mu \times 13.5 \mu$ \\
\hline Fixative (after 30 mins.) & $\ldots$ & $\ldots$ & $13.5 \mu \times 13.5 \mu$ \\
\hline $70 \%$ alcohol ... $\ldots$ & $\ldots$ & $\ldots$ & $13.5 \mu \times 13.5 \mu$ \\
\hline Paracarmine (20 mins.) & $\ldots$ & ... & \\
\hline Absolute alcohol $\quad \ldots$ & $\ldots$ & $\ldots$ & $13.5 \mu \times 13.5 \mu$ \\
\hline Xylol ... & $\ldots$ & $\ldots$ & $12.75 \mu \times 12 \cdot 75 \mu$ \\
\hline Balsam (immediately) & $\ldots$ & $\ldots$ & $12 \mu \quad \times 12 \mu$ \\
\hline Balsam (after $1 \frac{1}{2}$ hrs.) & $\ldots$ & ... & $\times 12 \mu$ \\
\hline
\end{tabular}


It will be seen that in this experiment the size of the cyst was unchanged by fixation, staining, and dehydration; but that a slight diminution in size was observed in xylol, and a further small reduction in balsam. This was clearly due to the circumstance that the cyst wall was still partly visible in the former, but completely invisible in the latter. The cyst wall remained unstained by the paracarmine; and there could be no doubt that the measurements of the "cyst" in balsam were really those of the stained protoplasmic contents only.

Expt. 2. (Cyst No. 7 of Table III).

\begin{tabular}{|c|c|c|c|}
\hline Medium in which cyst was examin & & & Dimensions \\
\hline Gelatine jelly (living cyst) & & ... & $12 \cdot 75 \mu \times 12 \mu$ \\
\hline Fixative (after 5 mins.) & $\ldots$ & $\ldots$ & $12.75 \mu \times 12 \mu$ \\
\hline Fixative (after $1 \frac{1}{2} \mathrm{hrs}$ ) & ... & ... & $12 \cdot 75 \mu \times 12 \mu$ \\
\hline $50 \%$ alcohol ... $\ldots$ & $\ldots$ & $\ldots$ & $12 \cdot 75 \mu \times 12 \mu$ \\
\hline Paracarmine (20 mins.) & $\ldots$ & ... & \\
\hline Absolute alcohol $\quad \ldots$ & $\ldots$ & $\ldots$ & $12.75 \mu \times 12 \mu$ \\
\hline Xylol ... $\quad \ldots$ & $\ldots$ & $\ldots$ & \\
\hline Balsam (immediately) & $\cdots$ & $\ldots$ & $10.5 \mu \times 9 \mu$ \\
\hline Balsam (after $1_{\frac{1}{2}}^{1}$ hours) & ... & $\ldots$ & $10 \cdot 5 \mu \times 9 \mu$ \\
\hline
\end{tabular}

Continuous observation of the cyst showed that the final decrease in size took place as follows. In absolute alcohol the protoplasm underwent a very slight shrinkage from the cyst wall. (The measurements in this medium are those of the cyst wall-not of the contents). In xylol considerable further contraction of the protoplasm was observed, a space separating the contents from the wall. In balsam the cyst wall, and consequently the space between it and the protoplasm, became completely invisible. The greatly reduced dimensions are therefore those of the shrunken protoplasmic contents only.

\section{Expt. 3. (Cyst No. 13 of Table III).}

In the two previous experiments the staining of the cyst was effected with paracarmine (in $70 \%$ alcohol) in order to avoid the tedium of transferring the cyst through the many grades of alcohol necessary in using a watery stain. We considered it desirable, however, to make further observations on cysts stained in haemalum, since this is the method we have employed as a routine throughout our investigations. We soon found, however, that the cysts were liable to drop out of the film when transferred to water, unless the gelatine was thoroughly 
hardened after fixation, and numerous grades of alcohol employed. In the following experiment, the gelatine was hardened in formol-alcohol (formol ( $40 \%$ formaldehyde) and absolute alcohol, equal parts). As will be seen this procedure had no visible effect on the dimensions of the cyst. Measurements were made in each grade of alcohol used.

\begin{tabular}{|c|c|c|c|c|c|}
\hline \multicolumn{4}{|l|}{ Medium in which cyst was examined } & \multicolumn{2}{|c|}{ Dimensions } \\
\hline Gelatine jelly (living cyst) & & & $\cdots$ & $14 \cdot 25 \mu$ & $\times 12.75 \mu$ \\
\hline Fixative (after 5 mins.) & $\ldots$ & $\ldots$ & $\ldots$ & $15 \mu$ & $\times 12 \mu$ \\
\hline Fixative (after 40 mins.) & $\ldots$ & $\ldots$ & $\ldots$ & $15 \mu$ & $\times 12 \mu$ \\
\hline Formol-alcohol (after $2 \frac{1}{2} \mathrm{hrs}$ & )$\ldots$ & $\ldots$ & $\ldots$ & $15 \mu$ & $\times 12 \mu$ \\
\hline Alcohols $35 \%, 20 \%, 10 \%$ & ... & $\ldots$ & $\ldots$ & $15 \mu$ & $\times 12 \mu$ \\
\hline Distilled water ... $\quad \ldots$ & $\ldots$ & $\ldots$ & $\ldots$ & $15 \mu$ & $\times 12 \mu$ \\
\hline Haemalum (15 mins.) ... & $\ldots$ & $\cdots$ & $\ldots$ & & \\
\hline Tap water $\quad \ldots \quad \ldots$ & $\ldots$ & $\ldots$ & $\ldots$ & $15 \mu$ & $\times 12 \mu$ \\
\hline Alcohols $10 \%, 20 \%, 35 \%$ & $0 \%$ & $0 \%$ & $\%$ & $15 \mu$ & $\times 12 \mu$ \\
\hline Absolute alcohol $\quad \ldots$ & $\ldots$ & $\cdots$ & $\ldots$ & $15 \mu$ & $\times 12 \mu$ \\
\hline Xylol $\quad \ldots \quad \ldots$ & $\ldots$ & $\ldots$ & $\ldots$ & $13 \cdot 5 \mu$ & $\times 10.5 \mu$ \\
\hline Balsam (after 20 mins.) & $\ldots$ & $\ldots$ & $\ldots$ & $13 \cdot 5 \mu$ & $\times 10.5 \mu$ \\
\hline
\end{tabular}

It will be apparent from the foregoing figures that the dimensions of the cyst underwent no change until its final transference to xylol and balsam. (There was a slight change in shape, but not in size, in the fixative.) Here again the final reduction in size was clearly seen to be due to the disappearance of the cyst wall in the clearing and mounting media.

It will be noticed that in Expts. 1 and 3 the actual loss in diameter observed was 1.5 longer, and of $3 \mu$ in the shorter diameter of the cyst. If the diminution in size had been due only to the disappearance of the cyst wall, there should have been only a loss of $1 \mu-1 \cdot 2 \mu$ in the diameters. In Expt. 2 the greater reduction was clearly due to the shrinkage of the protoplasmic contents; and it seems reasonable to suppose that the slightly greater loss than we expected in the other two experiments was due to the same cause. It will be remembered that in our earlier observations on the mean size of 500 cysts in saline solution and in balsam, we actually found a difference in average diameter of only about $1 \mu$. We think that this was probably due to the fact that the fixed cysts were very thoroughly hardened in alcohol before staining and mounting, in order to prevent shrinkage as far as possible: and, in measuring these cysts subsequently, we rejected all those which showed signs of shrinkage. 
In all the experiments hitherto described, we have confined our attention to cysts of large size (with diameters usually of about 12-14 $\mu$ ). Our conclusions, therefore, are directly applicable to cysts of these dimensions only. The difficulty of manipulating very small cysts, and the much greater difficulty of discounting errors in the measurement of objects of very small dimensions, deterred us from attempting to extend our observations to cysts of smaller size. It seemed to us almost certain, however, that closely comparable results would have been obtained for these. We have assumed, therefore, when dealing with the smallersized cysts of $E$. histolytica, that the effects of mounting in balsam would be the same, and the apparent reduction in size proportionate.

The foregoing experiments supply, we think, conclusive solutions of the problems which we set ourselves. We may therefore now summarize our results up to this point.

1. Cysts of $E$. histolytica which have been suitably fixed and stained and mounted in balsam, appear to possess a smaller mean diameter than those examined alive in saline solution, or after treatment with iodine. (There appears, however, to be no appreciable difference between the size of living cysts suspended in saline and those killed and suspended in Weigert's iodine solution.)

2. When properly fixed the cysts do not undergo any appreciable shrinkage in the fixative or in the usual reagents employed in staining and dehydration. The apparent loss of size takes place in the process of clearing and mounting; and is chiefly due to the disappearance of the cyst wall when the cyst is placed in a medium of high refractive index. Shrinkage of the protoplasmic contents from the cyst wall may also, in some cases, account for a still further diminution in the apparent diameter.

3. The average thickness of the cyst wall of $E$. histolytica was found to be $0.5 \mu$. In an ideal case, therefore, in which no protoplasmic shrinkage occurs, and the apparent diminution of the diameter of the mounted as compared with the living cyst is due only to the disappearance of the cyst wall in the mounting medium, the difference in diameter should be approximately $1 \mu$. Comparative measurements of living cysts and of those mounted in balsam with due precautions to prevent shrinkage and discount its effects, actually gave a difference of this magnitude in the mean diameters of the cysts. Shrinkage of the protoplasm may, however, lead to a considerable further reduction in size. (In one case directly observed under the microscope there was a reduction in the mean diameter of $2 \cdot 6 \mu$.) 
4. Assuming that the figures given above-which were obtained from a study of large cysts-are proportionately applicable to cysts of all sizes, we conclude as follows: When the processes of fixing, staining, and mounting are suitably conducted, so that protoplasmic contraction is avoided or reduced to a minimum, there is an apparent reduction of about $10 \%$ in the diameter of a cyst mounted in balsam as compared with its true diameter in the living condition.

Part II.

Demonstration of the existence of races of $\mathbf{E}$. histolytica which differ from one another in the size of the cysts which they produce.

In our earlier paper (1917) we briefly noted some results obtained in the study of cysts from over 200 patients infected with $E$. histolytica. We pointed out that there is a very wide range of variation in the size of the cysts passed by different persons; the extreme limits which we observed in this respect being represented by diameters of $5 \mu$ and $20 \mu$ for spherical cysts, examined alive in saline solution or killed and suspended in iodine. The measurements which we are now about to give were all made upon cysts which had been previously fixed, stained, and mounted in balsam. In comparing them, therefore, with our former figures it will be necessary to introduce the correction indicated in the preceding section of the present paper. The cases here described are, however, for the most part selected from the same series. So far as our technique is concerned, it will suffice to note that it has been uniform throughout, and is that described above. We must emphasize once more, however, that we have endeavoured as far as possible to eliminate the error due to protoplasmic shrinkage, by thorough hardening of the cysts before mounting them, and by omitting distorted or obviously shrunken cysts from our measurements. We believe, therefore, that the difference in the size of the cysts due to cytological manipulation approximates fairly closely to the ideal value to be expected from our preliminary investigations. Furthermore, as already noted, our conclusions are based upon measurements of 500 cysts in every case-250 measured by each of us independently.

Measurements of the cysts from one case (E. 42) in our series have already been given in some detail on an earlier page (see Table I). The cysts of this strain of $E$. histolytica are of comparatively large size, the summit of the curve plotted from them being at $12 \mu$ for the fixed and stained cysts. Strains such as this are, in our experience, the commonest. 
We find, however, that there are strains which produce cysts having a mean size either smaller or greater than this. Strains of much smaller size--the commonest being those with an average diameter of $7 \mu$ to $8 \mu-$ are not uncommon; but strains with a diameter considerably greater are by no means common. We have already (1917) noted that, in a series of over $200 \mathrm{E}$. histolytica infections which we studied, over $30 \%$ were infections with cysts of the smaller sizes. But we have seen so few infections with cysts having a mean diameter greater than $14 \mu$ that we are at present unable to form any accurate estimate of their real frequency.

In the following table (Table IV) we give the results of four series of measurements of the cysts from four different carriers of $E$. histolytica. The measurements, as before, are all given in units of the micrometer scale-not in microns. Case E. 42 has already been recorded (see Table I, line 3), but is repeated here for comparison with the others.

TABLE IV.

Measurements of cysts belonging to four different strains of $\mathbf{E}$. histolytica.

\begin{tabular}{|c|c|c|c|c|c|c|c|c|c|c|c|}
\hline \multirow{2}{*}{\multicolumn{2}{|c|}{ Case No. }} & \multicolumn{10}{|c|}{ Dimensions of cysts (in balsam) } \\
\hline & & $3 \cdot 0$ & $\mathbf{3 \cdot 5}$ & $4 \cdot 0$ & $4 \cdot 5$ & $5 \cdot 0$ & $5 \cdot 5$ & $6 \cdot 0$ & $6 \cdot 5$ & $7 \cdot 0$ & $7 \cdot 5$ \\
\hline H. 8. & ... & 8 & $\mathbf{3 3}$ & 194 & 140 & 102 & 19 & $\mathbf{3}$ & 1 & - & - \\
\hline H. 7 & $\ldots$ & 1 & 6 & 44 & 93 & 205 & 105 & 37 & 8 & 1 & - \\
\hline E. 42 & $\ldots$ & - & - & - & - & - & 1 & 0 & 9 & 60 & 90 \\
\hline \multirow[t]{2}{*}{ B. 1} & $\ldots$ & - & - & 一 & - & - & - & 1 & 1 & 11 & 18 \\
\hline & & \multicolumn{10}{|c|}{ Dimensions of cysts (in balsam) } \\
\hline Case No & & 8.0 & 8.5 & $9 \cdot 0$ & $9 \cdot 5$ & $10 \cdot 0$ & $10 \cdot 5$ & $11 \cdot 0$ & $11 \cdot 5$ & $\overline{12 \cdot 0}$ & 12.5 \\
\hline H. 8 & $\ldots$ & - & - & - & - & - & - & - & - & - & - \\
\hline H. 7 & $\ldots$ & - & - & - & - & - & - & - & - & - & - \\
\hline E. 42 & $\ldots$ & 165 & 101 & 54 & 12 & 8 & - & - & - & - & - \\
\hline B. 1 & $\ldots$ & 57 & 92 & 152 & 71 & 55 & 23 & 5 & 7 & 6 & 1 \\
\hline
\end{tabular}

This table displays very clearly the differences in the dimensions of the cysts in these four races. The differences are, however, seen in a still more striking manner in the figures ${ }^{1}$ reproduced in Plate VI, and in the curves plotted from the measurements. To facilitate comparison we have plotted the four series together in a single chart (Fig. 4). In this, as in the others, the units of measurement have been converted into microns. It will be seen that the summit of the curve for each race falls

1 A full explanation of these figures is given in the description of the plate. 
at a different point: and if we apply the correction indicated in Part I of this paper, we get the following figures for the diameters of the living cysts which occur with greatest frequency in each of these four races:

Case H. 8, greatest frequency $6 \cdot 0 \mu$, representing $6 \cdot 6 \mu$ for living cyst

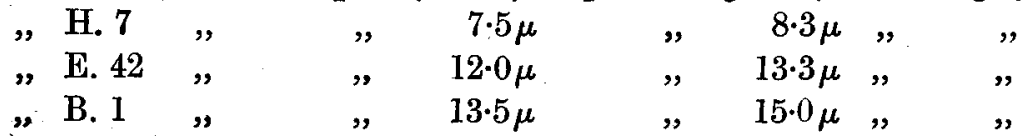

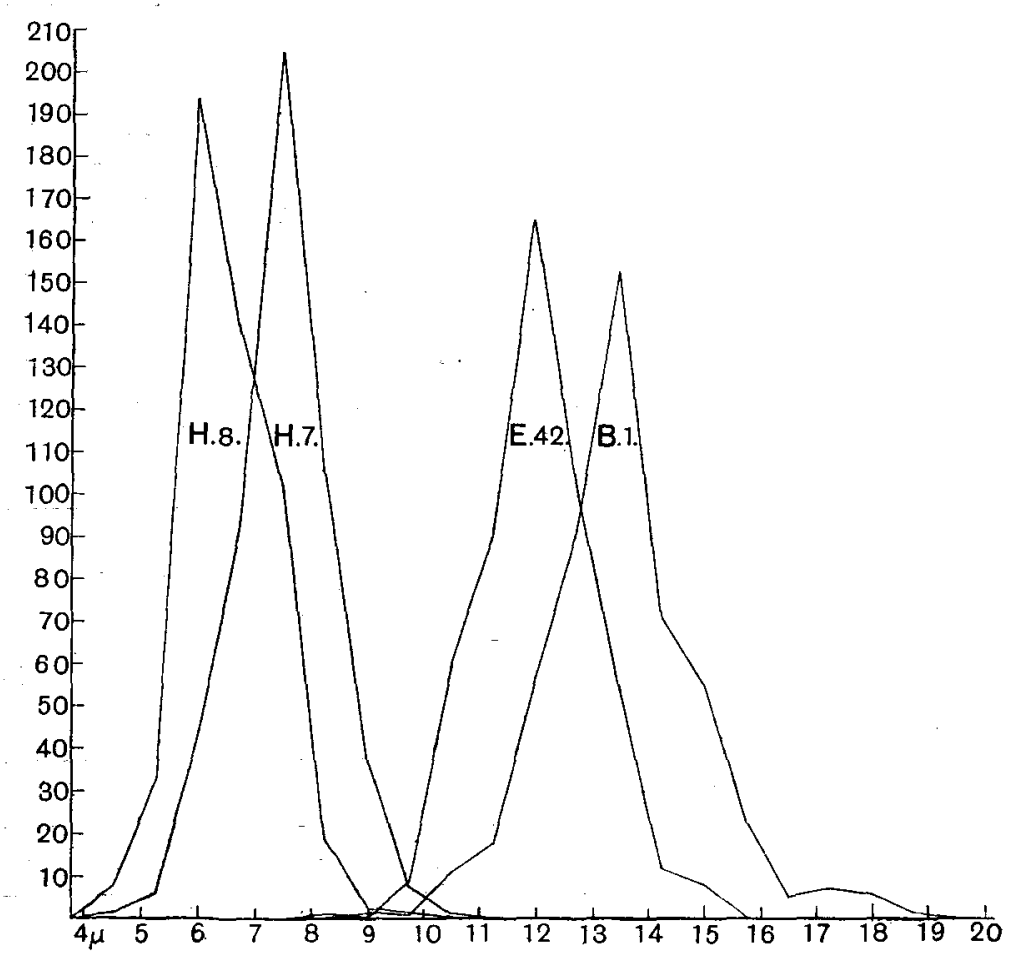

Fig. 4.

The figures calculated in this way for the living cysts were borne out by those which we had previously recorded after measuring a number of cysts in the fresh stools. These measurements were made during the ordinary routine examination of the stools of these patients, and are therefore based upon a comparatively small number of observations. Case H. 8, was recorded as having cysts about $6.5 \mu$ in diameter; Case H. 7, small cysts, variable in size, usually about $8-9 \mu$; Case E. 42 , cysts 
about 12-14 $\mu$; whilst Case B. 1 was merely noted as having many large cysts $15 \mu$ or more in diameter 1 .

Attention must here be drawn to the character of the curves obtained from each of our series of measurements. Inspection of Figs. 1-4 will convince the reader, we believe, that all the curves are of essentially the same form. They are unimodal, each with a single apex, and, like a normal curve of error, approximately symmetrical about the mode. With the exception of one curve (saline) shown in Fig. $2-$ which we have already discussed and concluded to be abnormal-we have up to the present obtained no curves which are not conformable to this type. We conclude from these findings that, in each case so far described, we are

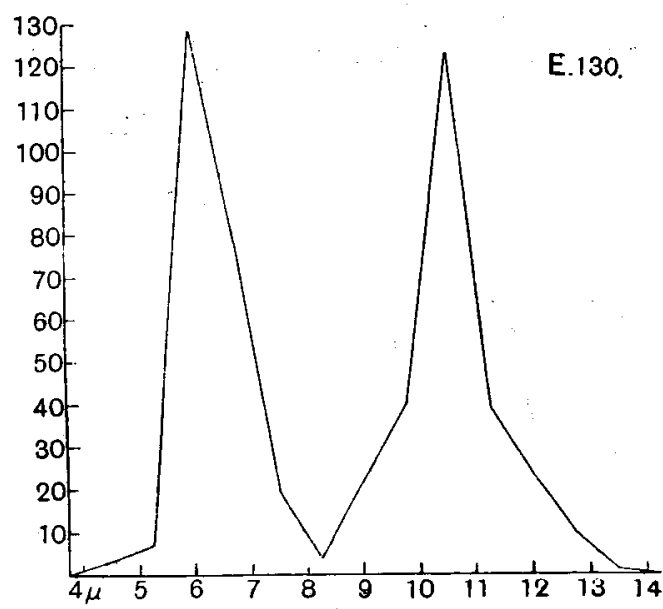

Fig. 5.

dealing with a single pure race of $E$ : histolytica producing cysts whose average diameter is constant, and which show merely those variations in size from the mean which are to be expected in any biological species or strain displaying the usual degree of variability.

For certain other cases, however, a different type of curve was obtained. Three such cases are shown in graphic form in Figs. $5^{2}, 6$ and 7 ; the actual measurements upon which these curves are based being given

${ }^{1}$ It is just possible that it may oceur to some reader that the large size of many of the cysts in the infections E. 42 and B. 1 is due to the inclusion of cysts of $E$. coli in the series measured. We would therefore state explicitly that neither of these cases is infected with this species, and that cysts containing more than four nuclei have never been found in the stools of either,

2 The cysts from this case (E. 130) were stained with Weigert's iron-haematoxylinnot with haemalum as were the others. 
in the following table (Table V). The measurements, as before, are given in units of the micrometer scale.

\section{TABLE V.}

Dimensions of cysts (in balsam)

$\begin{array}{lrrrrrrrrrrrrrrr}\text { Case No. } & & 3 \cdot 0 & \mathbf{3 \cdot 5} & \mathbf{4} \cdot 0 & \mathbf{4 \cdot 5} & \mathbf{5 \cdot 0} & \mathbf{5 \cdot 5} & \mathbf{6 \cdot 0} & \mathbf{6 \cdot 5} & \mathbf{7 \cdot 0} & \mathbf{7 \cdot 5} & \mathbf{8 \cdot 0} & \mathbf{8 \cdot 5} & \mathbf{9 \cdot 0} \\ \text { E. 130 } & \ldots & 3 & 7 & 131 & 78 & 19 & 4 & 22 & 40 & 123 & 39 & 23 & 10 & 1 \\ \text { E. } 79 & \ldots & - & 2 & 52 & 59 & 89 & 28 & 24 & 44 & 68 & 63 & 56 & 12 & 3 \\ \text { H. } 11 & \ldots & - & - & 7 & 16 & 12 & 3 & 35 & 86 & 160 & 97 & 64 & 18 & 2\end{array}$

The outstanding feature of these curves is that they all possess two apices. Case E. 130 (Fig. 5) is perhaps the most striking. The distribution of the cysts around the two modes $(6 \mu$ and $10.5 \mu)$ is remarkably

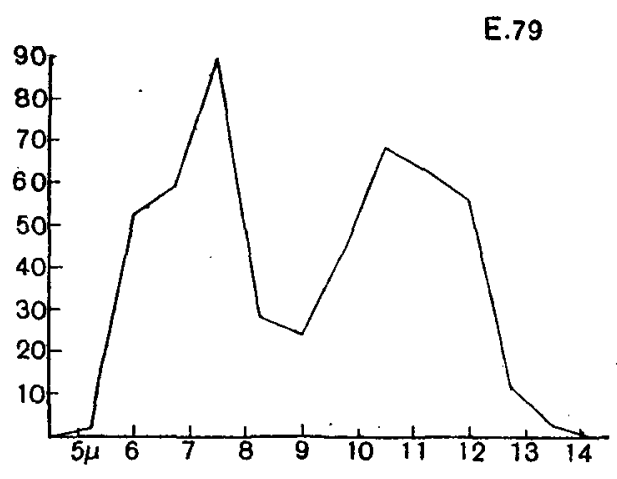

Fig. 6 .

symmetrical; and the entire curve may be compared with the two separate curves, taken together, from Cases H. 8 and E. 42 (shown in Fig. 4). The latter represented two different strains of $E$. histolyticafrom two different patients-and it can hardly be doubted that a similar interpretation must be applied to the former (Case E. 130): for here we obviously have an infection-in this case of the same patientwith two different strains simultaneously, the cysts belonging to each strain being present in approximately equal numbers. The other two cases (E. 79 and H. 11) give less symmetrical curves, but are obviously capable of like interpretation. Case H. 11, for example (see Fig. 7), clearly represents a double infection in which the number of cysts belonging to one strain (that producing large-sized cysts) greatly exceeds that of the other (that producing small cysts). 
The coexistence of two strains of $E$. histolytica in the same patientsuch, for example, as is seen in Case E. 130-is sometimes obvious from a mere inspection of a preparation containing the cysts. Nearly all appear to be either large or small, intermediates being either extremely scarce or apparently absent. In our earlier paper (1917) we noted the occurrence of two such double infections. (Case E. 130 is one of these.) But we also noted that infections occur in which cysts of large and small size with numerous intermediates are present; and we left it an open question whether such a case represents a multiple infection, or an infection with a single strain producing cysts of very variable size. Cases

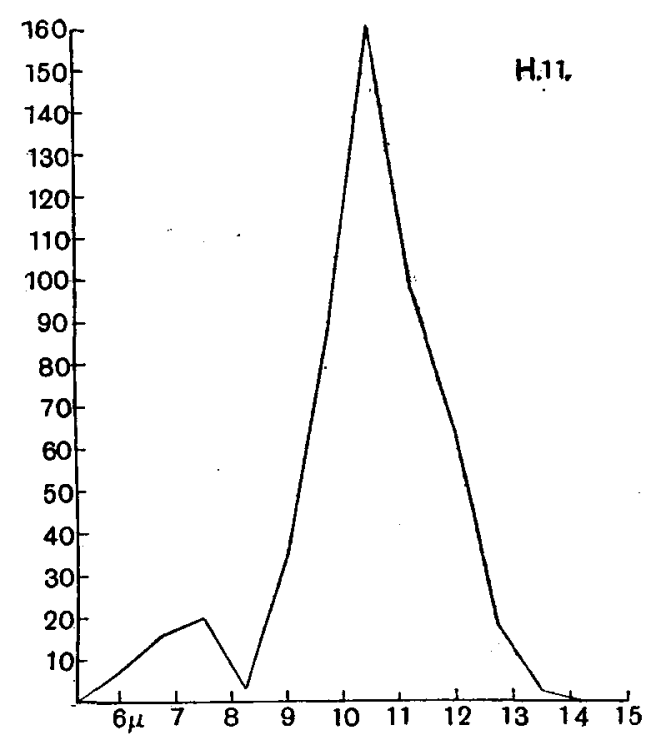

Fig. 7.

E. 79 and H. 11 are two such instances; and the curves in Figs. 9 and 10 appear to us to demonstrate the fact that each possesses in reality a double infection essentially similar to that of Case E. 130. Careful measurements, so far as they have been made, have not yet furnished us with one instance of a single strain showing such great variation in the size of its cysts. We believe, therefore, that when cysts showing a great range of variation in size (e.g. $7 \mu-15 \mu)$ are present in a stool, a careful series of measurements of a sufficiently large number will probably reveal the fact that they belong to two-or possibly more-different strains. 
-We have already given the calculated value for the commonest size of cyst occurring in each of the four simple infections which we have called H. 8, H. 7, E. 42, B. 1. If we deal in the same way with the three double infections just described, we obtain the following figures for the dimensions of the living cysts occurring with the greatest frequency:

$\begin{array}{cllll}\text { Case H. } 11 & \ldots & \ldots & 8 \cdot 3 \mu \text { and } 11.6 \mu \\ \Rightarrow & \text { E. } 79 & \ldots & \ldots & 8.3 \mu \text { and } 11 \cdot 6 \mu \\ , & \text { E. } 130 & \ldots & \ldots & 6.6 \mu \text { and } 11.6 \mu\end{array}$

On comparing these figures with those already given it will be seen that the smaller cysts in Cases H. 11 and E. 79 have the same diameter $(8 \cdot 3 \mu)$ as those of Case H. 7 ; whereas the smaller cysts of Case E. 130 correspond in size $(6 \cdot 6 \mu)$ to those of Case H. 8 . The larger strains from all these cases are, however, though coinciding with one another, different in size $(11 \cdot 6 \mu)$ from any of those which we have previously described. It appears, therefore, that the three double infections just described supply us with but one additional race. And hence we conclude that, in the stools of the seven patients studied, cysts belonging to five different races of $E$. histolytica were present. The approximate size of the living cysts occurring with the greatest frequency in each of these five races is as follows:

$$
6 \cdot 6 \mu, 8 \cdot 3 \mu, 11 \cdot 6 \mu, 13 \cdot 3 \mu, 15 \mu .
$$

It must be expressly noted that the strains of $E$. histolytica just described constitute merely a selection from those which we have studied. We have no doubt that, had it been possible for us to make an equally detailed study of all the cases in our series, we should have found other races possessing cysts with other mean diameters. As already noted above, we have found the strains with cysts having diameters from about $11 \mu$ to $14 \mu$ to be the commonest, though strains resembling in size those of Cases H. 8 and H. 7 are by no means infrequent. We have seen, however, very few strains indeed having mean diameters, for the living cysts, of intermediate magnitude (about $10 \mu$ ). Of the $202 \mathrm{E}$. histolytica infections which we described in our earlier note (1917) there was but one of this character (Case E. 154) ${ }^{1}$. A case which appears to be of

..1 Unfortunately, we have been unable to make a large series of measurements of the cysts from this case. When the case was first examined, the size of the cysts-based on relatively few measurements-was recorded, and stained preparations were made for further study. On examining these later, however, we were disappointed to find that the cysts were too distorted for satisfactory measurements to be obtainable. (They were fixed in Schaudinn's fluid-without acetic acid.) 
the same type has been recorded by Wenyon and O'Connor (1917Case "Kettlewell," p. 160). Of the other cases described by these observers, we note that one is similar to our Case H. 7; three others resemble our Case E. 42; and the remaining one is of the type of our Case B. 1. The figures, as far as they go, of these authors thus agree closely with our own; and they have also concluded that "it may be safely stated that the majority of cases of $E$. histolytica infection show cysts with a diameter of ten to fourteen microns." At the present time, however, we are not so much concerned with an investigation of the actual number of strains of $E$. histolytica which may exist, or of their frequency, as with the demonstration that such strains actually do exist.

For the complete demonstration of this fact, however, it is necessary to prove that the mean diameter of the cysts from any patient is not subject to any considerable variation from day to day, but remains constant. That this is so we think nobody who has studied any number of $E$. histolytica infections for any length of time will be inclined to doubt. It is, indeed, a fact which one confirms almost daily in the course of routine examinations. A patient originally found to be passing cysts of a certain mean diameter is found later to be passing cysts of the same size whenever his stools are positive; and this applies both to untreated cases, and also to those who relapse after treatment with emetine. Case E. 42, for example, who was under observation for about seven and a half months, during which time he was treated and relapsed four times, always, when positive, passed cysts similar to those already described (mostly 12-14 $\mu$ in diameter). We did not always measure a large number of cysts from his stools, but the measurements which we made were always closely similar. The two largest series of measurements of living cysts from this patient have already been recorded. Five hundred measured on May 21st, 1917, gave a mean diameter of approximately $13 \mu$, five hundred measured on June 23rd, 1917, after a prolonged course of unsuccessful treatment during the period between these dates, were found to have a mean diameter of $13 \cdot 2 \mu$. Similarly another case (E. 149) which was originally found to be passing cysts $6.5 \mu-8 \mu$ in diameter on January 16 th, 1917, remained under observation until the end of May. During this period he was unsuccessfully treated with emetine three times, and at each relapse cysts of the same size were present in his stools. Observations similar to the foregoing could be multiplied almost indefinitely: from our records. In fact it is a rule, to which so far we have found no exception, that the size of the cysts passed by any individual infected with $E$. histolytica remains constant as long as the infection persists. 
It is to be noted, however, that a patient infected with two different strains at the same time may present an apparent exception.to this rule. As is now well known, cysts are not always present in the stool of an infected person -or, at all events, are not always present in discoverable numbers. From time to time the stools are negative. Now it is clear that a person infected with two different strains may sometimes be negative for either or both. If, therefore, at a single examination cysts of large size are present, it cannot be concluded that the patient is infected with this strain only. At the next or at a later examination cysts of small size may be found, either alone or accompanied by the larger cysts. A careful study of such a case by more prolonged examination and the measurement of a large series of cysts will alone enable one to arrive at the correct interpretation. One of our cases (E. 58) furnishes us with a case in point. This man, when first examined, was found to be passing numerous cysts of $E$. histolytica of large size-mostly from $12 \mu-14 \mu$ in diameter. A second specimen, examined at a later date, contained cysts which at first sight appeared to be all of small size $(8 \mu-9 \mu)$. A careful study of this stool, however, revealed the fact that large-sized cysts were also present in very small numbers; and a series of measurements clearly showed that we were dealing with a double infection similar to those which we have already described. The diameters of the two strains were $8 \mu-9 \mu$ and $12 \mu-14 \mu$. Cases such as this, therefore, though apparently indicating at first sight an inconstancy in the size of the cysts, in reality, when carefully studied, furnish most conclusive evidence of the constancy of the different strains of $\boldsymbol{E}$. histolytica.

We believe, however, that certain conditions may, to a slight extent, temporarily modify the size of the cysts of a given strain. Although we can adduce no large series of measurements in support of our belief, we have often observed a slight increase in the size of the cysts from a patient just relapsing after unsuccessful treatment with emetine. These cysts are usually very degenerate, and frequently all uninucleate. In the case of the larger strains $(12 \mu-14 \mu$ before treatment) they may be $15 \mu$ or more in diameter; such large cysts constituting the great majority - not, as originally, the rare exceptions-among the cysts in the stool. These large and degenerate cysts often give rise to considerable diffculties or uncertainties in diagnosis. We have invariably found, however, that, after a few days, cysts of normal size and structure reappear in the stools; thus enabling one not only to confirm the diagnosis, but also to verify the fact that the administration of emetine has brought about no permanent change in the size of the cysts. 
Since it appears probable, from these observations, that a change in the environment of the amoebae-in the above case, a change produced by the administration of emetine to their host-may modify the size of the cysts which they produce, the suggestion might be made that the differences in size in the cysts from different patients may be due to comparable causes. It might be supposed, for example, that when one patient passes cysts with a diameter of $7 \mu$, whilst another passes cysts of $12 \mu$, this is not because they are infected with two different strains, but because the same strain, when living in two different environments (hosts), produces cysts of two different sizes. Unless such a possibility could be excluded, it is clear that we should not be completely justified in speaking of the different-sized cysts, in the infections which we have studied, as belonging to different strains or races of the parasite. It has not been possible to test this supposition by direct experimental infection of human beings by means of cysts of known sizes; but the question can nevertheless be answered almost conclusively, we believe, from some of the observations which we have already recorded. We think that we have demonstrated above that cysts of two different mean sizes may coexist in the stools of the same host (cp. Cases E. 130, E. 79, H. 11, supra); and this fact clearly indicates that the size of the cysts, whether large or small, is not determined by the host. It is of course conceivable, though we think highly improbable, that the site of infection in the large intestine might influence the size of the cysts produced by the parasites. But since we know no facts or analogies to support such an assumption it seems unnecessary to discuss it further. It seems to us, therefore, that we are justified in concluding ( 1 ) that the differences in mean diameter of the cysts passed by different persons are to be correlated with differences in the parasites themselves and not in their hosts; and (2) that these differences indicate that there are distinct groups of individuals among the parasites themselves which may properly be designated "strains" or "races" within the single species $E$. histolytica.

In concluding this section we may refer to a point of some practical importance connected with the recognition of the different strains of $E$. histolytica. It has been our custom for a considerable time to record the diameter of the cysts for every infection which we have studied. We have thus known with some degree of certainty with what strain or strains of $E$. histolytica each patient has been infected before beginning treatment with emetine. This knowledge has on several occasions proved useful subsequently. In dealing with a large number of military patients it is often difficult, and sometimes impossible, to make certain 
that every sample of faeces arriving at the laboratory has been passed by the particular patient whose name it bears. In spite of every precaution, substitution and interchange of specimens from time to time occur; and a case which is in reality negative may thus appear positive, and vice versa. On three occasions our patients have "relapsed" after treatment with emetine bismuth iodide, the "relapse" strain having cysts of a different size from those of the original infection. As such an occurrence appeared to us highly improbable in the light of the results given above, we were led on each occasion to challenge the authenticity of the specimens. A more rigorous control of the patients, and the continued examination of their faeces, ultimately proved in each case that our suspicions were justified. All three cases were subsequently found consistently negative, and were therefore finally discharged as cured. Had we not carefully studied and recorded the size of their cysts before treatment, each of these cases would, of course, have been wrongly classified as a relapse and re-treated.

COMPARISON OF OUR CONCLUSIONS WITH THOSE OF SOME OTHER WORKERS.

Before discussing our results and their relation to those of certain other observers, it will be convenient rapidly to survey these results themselves. We have, indeed, already summarized them to some extent in our previous paper (1917), and at various points in the course of the present work. But it will greatly facilitate the ensuing comparison if we again summarize our chief conclusions at this point collectively.

Our chief result is that we have, as we believe, demonstrated that the human dysentery amoeba (E. histolytica Schaudinn) is a collective species, which includes a number of distinct races or strains of individuals which can be readily distinguished from one another by the sizes of the cysts which they produce. The cysts produced by the different strains differ from one another morphologically in no respect save size, being in all other respects indistinguishable. Whether these strains differ from one another in pathogenicity or in other physiological characters we have as yet no means of determining with certainty. Nevertheless, we have observed that all react in the same way towards treatment with emetine, and we think it probable that no such differences exist. We believe that we have been able to show with considerable probability that the different groups of individuals constituting these so-called "strains" really are such, since they appear to be permanent and not to be merely a result of differing environmental conditions. In other words we regard them as constituting so-called "petites espèces" (Jordan) or "pure lines" 
(Johannsen) such as have been demonstrated to exist in almost all species of organisms which have hitherto been studied systematically'.

We have obtained no evidence that races of $E$. histolytica producing cysts of any particular size are characteristic of infections acquired in any particular locality. We have found cysts of the commonest size $(11 \mu-14 \mu)$ in carriers who contracted dysentery in all parts of the world from which we have examined cases (Europe, Asia, Africa, and America). The strains producing small cysts have been detected similarly in cases from many parts of the world (France, Egypt, Gallipoli, Salonica, Mesopotamia, India). Cysts of the larger diameters have been found in the stools of dysentery patients from Egypt, Gallipoli, Salonica, China, Thibet, Malay States, and S. Africa. It is not of course possible to say that the infection was acquired in every case when and where the attack of dysentery occurred, though this appears probable in many cases.

We have pointed out elsewhere (1917) that the name "E. minuta" is improperly applied to those races of $E$. histolytica which produce cysts of small size. We think it necessary to emphasize this once more, since the contrary has again been asserted by Woodcock (1917). He says "the name 'minuta' should be reserved for the distinctly small type or variety, the cysts of which are only about 8 microns in diameter." There can be no question however that the name Entamoeba minuta was given by Elmassian (1909) to the common race of $E$. histolytica producing cysts of large size (about $12 \mu$ in diameter). It follows, therefore, that minuta is a synonym without validity for histolytica; and if the name "minuta" is retained for any stage in the development of this parasite, then it must be applied only to the small precystic amoebae, characteristic of subacute infections, which were described and figured by Elmassian and to which he gave the name.

1 Comparable instances in the Protozoa are to be seen in the two species Paramecium caudatum and $P$. aurelia. It has been shown by Jennings (1908) that within each of these species there are numerous strains which differ from one another only in the average size of their constituent individuals. It is true that in this case the characters distinguishing the pure lines from one another are those of the organisms themselves-not of their cysts. In the case of $E$. histolyticn we are still uncertain whether the full-grown amoebae belonging to strains producing cysts of different size are themselves likewise distinguishable in the same respect. (The precystic "minuta" forms of the different strains undoubtedly correspond in size to the sizes of cysts which they produce. Cp. Plate VI, Figs. $E . h$. (2) and $E$. $h$. (3), and see also our earlier paper, 1917.). Nevertheless, whether this be the case or not appears to us immaterial, since the size of the individual or the size of the cyst might be used with equal propriety to discriminate races from one another. A summary of the literature bearing upon pure lines in the ciliate Protozoa has been published elsewhere by one of us (C. D. 1914). 
It is of course possible to regard each race possessing cysts of a given size as a distinct variety which merits a distinctive varietal name, assigned in accordance with the rules of nomenclature. At present, however, it seems to us superfluous to invent such names, since we do not know how many different races actually exist, and since for those races already studied a simple numerical expression of the size of the cysts furnishes an adequate distinguishing mark.

From a practical point of view it is of course most important that the cysts of the various strains of $E$. histolytica should be recognized and accurately distinguished from those of the other amoebic parasites of the human bowel. This aspect of the matter has, however, already been considered by Wenyon and O'Connor (1917) and ourselves (1917), and need not be further discussed here.

As we have already noted more than once, our conclusions in general appear to agree very closely with those of Wenyon and O'Connor (1917); but this is by no means the case when we come to consider the results of some other workers who have published figures and statistics relating to the cysts of $E$. histolytica. Two such publications we shall consider in some detail.

1. Kuenen and Swellengrebel (1913). These authors have stated that the cysts of $E$. histolytica vary in size from $11 \mu$ to $19 \mu$. They give a curve which possesses a single apex situated at $13 \mu$. With regard to these findings we.will merely note the following points. First, the form of the curve obtained does not differ in any important respect from some of our own. Secondly, it seems clear that their figures apply, not to the whole species $E$. histolytica, but only to one of the commoner strains producing large cysts-the strains producing small cysts having escaped the authors' notice completely. And thirdly, it is clear that no farreaching conclusions can be drawn from the study of a single strain, and of only a hundred cysts belonging to it-the number actually considered by these observers. Although at first sight, therefore, these results may appear very different from our own, we believe that they become easily reconcilable with them when the deficiencies just noted are taken into account.

2. Mathis and Mercier $(1916,1917)$..-In a series of papers these two authors have recently made and repeated a number of statements concerning the diameters of the cysts of E. histolytica; and have moreover drawn from their findings certain conclusions concerning the life cycle of the parasite. Their conclusions are briefly as follows (see especially Mathis and Mercier, $1916 a$, p. 980, and $1917 a$ ): 
1. The cysts of $E$. histolytica possess diameters ranging from $10 \mu$ to $15 \mu$ (living cysts). They never pass these limits.

2. The majority of the cysts are either $12.5 \mu$ or $14.0 \mu$ in diameter. From $40 \%$ to $45 \%$ of the cysts in every infection fall under each of these categories-larger, smaller, and intermediate-sized cysts being exceptional.

3. The cyst population is thus dimorphic, consisting of "microcysts" and "macrocysts." It is supposed that, on being ingested by a fresh host, they liberate respectively microgametes and macrogametes.

Before considering these conclusions in detail we will briefly refer to the methods of investigation employed by these authors, whose results are so conspicuously different from our own. It is clear, both on general grounds and for particular reasons considered in the earlier part of the present paper, that, to obtain satisfactory results in an investigation of the present character, the greatest attention must be paid to methods and technique. It is regrettable, therefore, that the authors have not thought it necessary to give anything but the slightest indications in this respect. They tell us merely that their measurements have been made-they do not tell us how-on stained and mounted cysts; that with the technique which they employed (fixation with sublimatealcohol-acetic acid, staining with iron-haematoxylin and eosin) the cysts undergo a contraction of $2 \mu$ to $2.5 \mu$-for which a correction is made to determine the size of the living cysts; and, though no adequate figures are given, that the dimorphism can be made evident by measuring 200 cysts. The only curve which they give (1917 $a$ ) in illustration is, however, based upon the measurements of only one hundred.

Since the authors have not yet described (so far as we are aware) their methods of measuring cysts-which, as we have seen, is a by no means simple matter-it is impossible for us to judge whether the discrepancy between their findings and ours depends upon differences in the technique adopted. This lack of information becomes especially baffling when we come to consider the figures which they give for the differences in size between living cysts and those which have been fixed, stained, and mounted. The authors' method of fixation was similar to our own; and their method of staining sufficiently like ours to make it probable that it could not account for any considerable difference in the results. Their statement, however, that the process of fixation, staining, and mounting causes a cyst to undergo a reduction of $2 \mu$ to $2.5 \mu$ in diameter is completely opposed to our findings. Since we have devoted much attention to this matter, and all our findings have been perfectly con- 
sistent, we can only suppose that the authors are mistaken in their assertion; and that, in the absence of any indication to the contrary, we are justified in concluding that the lightness with which they dismiss this difficult problem reflects the spirit in which they effected its solution.

It seems clear, from their published statements, that Mathis and Mercier have, like Kuenen and Swellengrebel, ignored all strains of E. histolytica save the commonest-those which, as in our Case E. 42 , produce cysts having a mean diameter between $12 \mu$ and $14 \mu$. The smaller and larger strains they have, apparently, completely overlooked.

The curve which Mathis and Mercier have published (1917 a, p. 168), and upon which the proof of a dimorphism in the cysts of $E$. histolytica appears to rest, is to us wholly incomprehensible. As we have seen, the measurements which we have made of single strains of $E$. histolytica have consistently given us unimodal curves. There has always been a common diameter for the majority of the cysts, with the minority grouped more or less symmetrically above and below it. Even by taking so few as a hundred cysts we cannot understand how it has been possible to obtain two modes separated from one another by only $1.5 \mu$, with only half-a-dozen cysts of intermediate dimensions. In no instance have a hundred cysts taken at random from our series of measurements given us a curve of this form. We were at first inclined to suppose that the dimorphism was similar to that which we ourselves have found in Case E. 130-that is to say, it resulted from an infection with two different strains. This explanation, however, will not suffice: for the superposition of the curves from two different strains having their maxima at $10 \mu$ and $11.5 \mu$ would not give a curve like that of Mathis and Mercier. It would be necessary to assume in addition that the strain having the larger cysts showed practically no variation in size below the mean, while the smaller showed none above. Another possible explanation is that which we have suggested (supra, Part I, A) to account for the anomalous bimodal curve which we obtained from one of our own series of measurements.

Whatever the correct interpretation may be, it is clear from our own measurements that there are many strains of $E$. histolytica whose cysts display no discoverable dimorphism in respect of size. Indeed, it appears to us that when a dimorphism of the cysts in any given stool is demonstrable, it is to be interpreted as the result of a simultaneous infection of the patient with two different strains. This very obvious interpretation is not even considered by Mathis and Mercier, though no proof that there is a dimorphism of the cysts in any-still less in every-strain can be furnished until this possibility has been excluded. For our own 
part we can only suppose, in the light of our own results, that the conclusions of Mathis and Mercier rest upon some fallacy in observation or interpretation which, in the absence of all data for its determination, we are at the moment unable to define.

Although we believe that the conclusion of Mathis and Mercier that the cysts of $E$. histolytica are constantly differentiated into "microcysts" and "macrocysts," destined to liberate microgametes and macrogametes respectively, is unsupported by facts, we would not be understood to maintain, either that the small amoebae which emerge from the cysts are not gametes, or, if they are, that they do not belong to two different categories. In the absence of any evidence save a somewhat vague analogy with other organisms, we think it is as yet unprofitable to discuss such hypotheses further. We may, however, point out that, far from denying a sexual development in $E$. histolytica at the stage in the lifehistory and of the kind postulated above, this very suggestion was, we believe, made originally by one of us (C. D., 1909) as a result of a detailed study of the very closely related species $E$. ranarum.

\section{SUMmary AND CHIEF CONClUSions.}

1. Entamoeba histolytica Schaudinn (vel E. dysenteriae Councilman and Lafleur), the human dysentery amoeba, is a collective species. It comprises a number of distinct races, strains, or pure lines, distinguishable from one another by the size of the cysts which they produce.

2. How many such distinct races exist is still undetermined; but we have demonstrated the existence of at least five.

3. There is no evidence that the different races differ in their geographical distribution, or in any character save size.

4. These races remain constant in character within a given host; and the dimensions of the cysts are not determined by the action of the host upon the parasite, since two different races may coexist side by side in the same host.

5. Different values for the dimensions of the cysts are obtained when these are measured in different media. Taking the measurements of living cysts suspended in physiological saline solution as representing the true value, we find that the dimensions of cysts in iodine solution are approximately the same; but that cysts which have been suitably fixed, stained, and mounted in balsam, have undergone an apparent reduction of approximately $10 \%$ from the true diameter.

6. The most suitable fixative for $E$. histolytica cysts we have found to be a modification of Schaudinn's fluid. (Saturated aqueous solution of 
corrosive sublimate, two parts; absolute alcohol, one part; glacial acetic acid, 4-5\%.) Cysts fixed in this fluid undergo no measurable shrinkage or swelling in the process.

7. The apparent loss of size observed in cysts which have been fixed, stained, and mounted in balsam, is due chiefly to the invisibility of the cyst wall in the mounting medium.

8. It follows from what has been said above ( 5 and 6 ) that to obtain an approximately true value for the dimensions of the living cysts from measurements made upon those suitably fixed, stained, and mounted in balsam, these measurements should be multiplied by the factor $\frac{10}{9}$.

9. We find no evidence of the existence of a sexual dimorphism in the cysts belonging to a single race, such as has been described by Mathis and Mercier.

10. Since cysts of $E$. histolytica may be found with all diameters from about $5 \mu$ to about $20 \mu-$ a range overlapping or covering the dimensions of the cysts of the two common harmless intestinal amoebae of man ( $E$. coli and $E$. nana) and of other cysts and cyst-like bodies occurring in human faeces-it is of considerable practical importance to recognize and distinguish the diverse races of $E$. histolytica which differ from one another in the dimensions of the cysts which they produce.

\section{REFERENCES.}

DobelL, C. (1909). Researches on the intestinal Protozoa of frogs and toads. Quart. Journ. Micro. Sci. LmI. 201.

- (1914). A commentary on the genetics of the ciliate Protozoa. Journ. Genet. IV. 131.

- (1. 1918). Are Entamoeba histolytica and Entamoeba ranarum the same species? An experimental inquiry. Parasitology, x. 294.

DobeLL, C. and JEPPS, M. W. (1917). On the three common intestinal entamoebae of man, and their differential diagnosis. British Med. Journ. II. 607.

Elmassian, M. (1909). Sur une nouvelle espèce amibienne chez l'homme, Ent. amoeba minuta n. sp. Centralbl. f. Bakt. I. (Orig.) LIr. 335.

Jennings, H. S. (1908). Heredity, variation and evolution in Protozoa. II. Proc. American Phil. Soc. XLVII. 393.

Kuenen, W. A. and Swellengrebel, N. H. (1913). Die Entamöben des Menschen und ihre praktische Bedeutung. Centralbl. f. Bakt. I. Abt. (Orig). LXxI. 378.

Mathis, C. and Mercier, L. (1916). L'amibe de la dysenterie. Bull. Inst. Pasteur, xiv. 641 .

(1916 a). Les kystes d'Entamoeba dysenteriae. C. R. Soc. Biol. Paris. LXxrx. 980.

(1917). Identification des kystes des entamibes intestinales de l'homme. Presse Medicale, No. 12. 
PARASITOLOGY, VOL: X. NO. 3

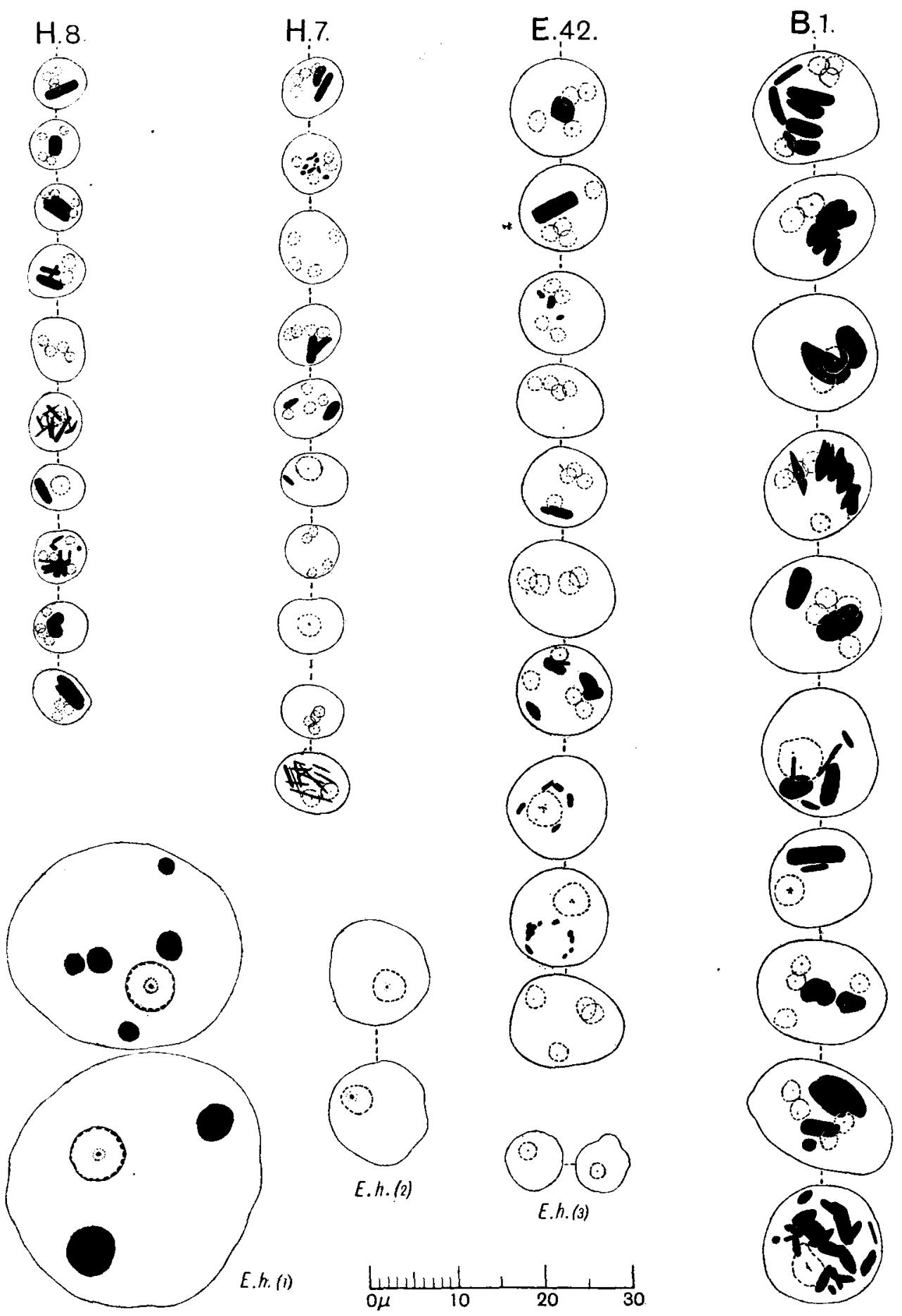

C. D. del. 
Mathis, C. and Mercier, L. (1917 a). Existe-t-il des kystes à plus de quatre noyaux chez Entamoeba dysenteriae? Bull. Soc. Pathol. Exot. x. 165.

- (1917 b). La schizogonie chez les entamibes intestinales de l'homme. Ibid. 311.

Wenyon, C. M. and O'Connor, F. W. (1917). An inquiry into some problems affecting the spread and incidence of intestinal protozoal infections, etc. Part II. Journ. Royal Army Med. Corps, xxvir. 151.

Woopcock, H. M. (1917). Protozoological experiences during the summer and autumn of 1916. Ibid. xxIx. 290.

\section{DESCRIPTION OF PLATE VI.}

The figures in this plate have been drawn to show at a glance the differences in the dimensions of the cysts of $E$. histolytica in the four cases (H. 8, H. 7, E. 42, B. 1) described in the text. They show in parallel columns ten cysts from each of these cases. The cysts were not selected, but represent in each case ten consecutive cysts in the order in which they appeared in examining the preparations at random under the microscope. The preparations were all fixed, stained, and mounted in the manner described in the text. The drawings show merely the outlines of the cysts, their nuclei, and chromatoid bodies (black) when present. The outlines were drawn as accurately as possible with the camera lucida, at a magnification of 2500 diameters (Zeiss $2 \mathrm{~mm}$. apochromat, N.A. $=1 \cdot 40$, compensating ocular (8), and have been reduced to the size shown in the process of reproduction. The figures illustrate not only the differences in the dimensions of the eysts in the four races of $E$. histolytica described, but also the kind of asymmetry observable in the outlines of the cysts themselves, which renders a perfectly accurate determination of their diameters. extremely difficult.

The remaining figures are outlines of fixed and stained amoebae, drawn in the same way to the same scale, to illustrate the size of the unencysted organisms as compared with their cysts. Fig. $E . h$. (1) shows two individuals of the tissue-inhabiting form ("histolytica" form) of $E$. histolytica, from a case of acute dysentery. The amoebae belong to a race producing cysts similar in size to those of Case E. 42. Fig. $E$. $h$. (2) shows two small precystic amoebae ("minuta" forms) from a subacute infection, and belonging also to a race of $E$. histolytica having cysts of similar size. Fig. $E$. $h$. (3) shows a pair of precystic amoebae ("minuta" forms) belonging to a race with cysts similar in size to those of Case H. 8. (The black masses in Fig. $E$. $h$. (1) are the remains of ingested red blood-corpuscles.) The amoebae were not specially selected individuals ${ }_{5}$ but were taken at random from preparations in which large numbers were present. 